\title{
Die Reform der Strafbarkeit von Menschen- und Frauenhandel aus kriminologischer Sicht
}

\section{Ziele der Änderungsgesetzgebungen}

$\int$ ie Straftatbestände der Vermittlung in oder der Förderung der Prostitution wurden in den letzten 15 Jahren mehrfach geändert. Erstmals 1993 wurde Frauenhandel in Umsetzung einer EU-Richtlinie zu einem eigenständigen Delikt mit einem für Sexualdelikte ungewöhnlichen Rechtsgut, nämlich der Freiheit der sozialen und wirtschaftlichen Betätigung. 2002 wurde im Zuge der Legalisierung der freiwilligen Prostitution von Erwachsenen die bloße »Förderung « entkriminalisiert (das Verbot war ohnehin ein reiner Ermittlungsparagraph gewesen) und nur noch die ausbeuterische Prostitution verboten. Strafrechtlich war dieser Begriff bis dahin nur im Zusammenhang mit der Zuhälterei bekannt und deshalb sehr eng ausgelegt. Aber was dies nun strafrechtlich und - was $\mathrm{m}$. E. wichtiger ist - zivilrechtlich bedeutet, blieb damals im dunklen und wurde auch in der Folgezeit nicht gerichtlich präzisiert. Die Fixierung auf das Strafrecht verhinderte Standards für angemessene Löhne und gewerbliche Mieten. Hinzu kam eine Schwäche der professionellen Beratung der Betroffenen, welche Zivilverfahren vermied und damit eine Klärung, welche Verträge und Absprachen (auch faktische Arbeitsverhält-

nisse) gegen das Verbot der "Ausbeutung « verstoßen und deshalb nichtig sind (§ 134 BGB). Offenbar dachten diejenigen, welche das Prost $\mathrm{G}$ hätten aktiv umsetzen müssen, mehr an den Ausstieg aus als die rechtliche Regulierung der Branche und versäumten es, die sozialen und rechtlichen Rahmenbedingungen zu schaffen, um Bordellbetreiber zu zwingen, Steuern und Sozialabgaben abzuführen. Auch der Versuch von Verdi über eine gewerkschaftliche Unterstützung nachzudenken blieb vereinzelt und folgenlos. Das Recht des sexuellen Dienstleistungsgewerbes muss folglich erst noch geschrieben werden. Zwar gibt es einen juristischen Kommentar von Margarete von Galen, aber keine weiterführende Diskussion zu den dort gegebenen Anregungen. Stattdessen dominieren ganz enge ausländerrechtliche Lesarten und eine notorische (und unerfüllbare) Klage über unzureichende Befugnisnormen für die Polizei.

\section{Zwangsprostitution - Was zwingt Menschen in die Prostitution?}

Deklarierte Opfer und ermittelte Tatverdächtige betragen - mit Schwankungen - etwa 800-1200 Personen. Verurteilungen sind aber selten, 2002

Phänomenologie des polizeilichen Hellfeldes: die deklarierten Opfer von Menschenhandel nach Herkunft

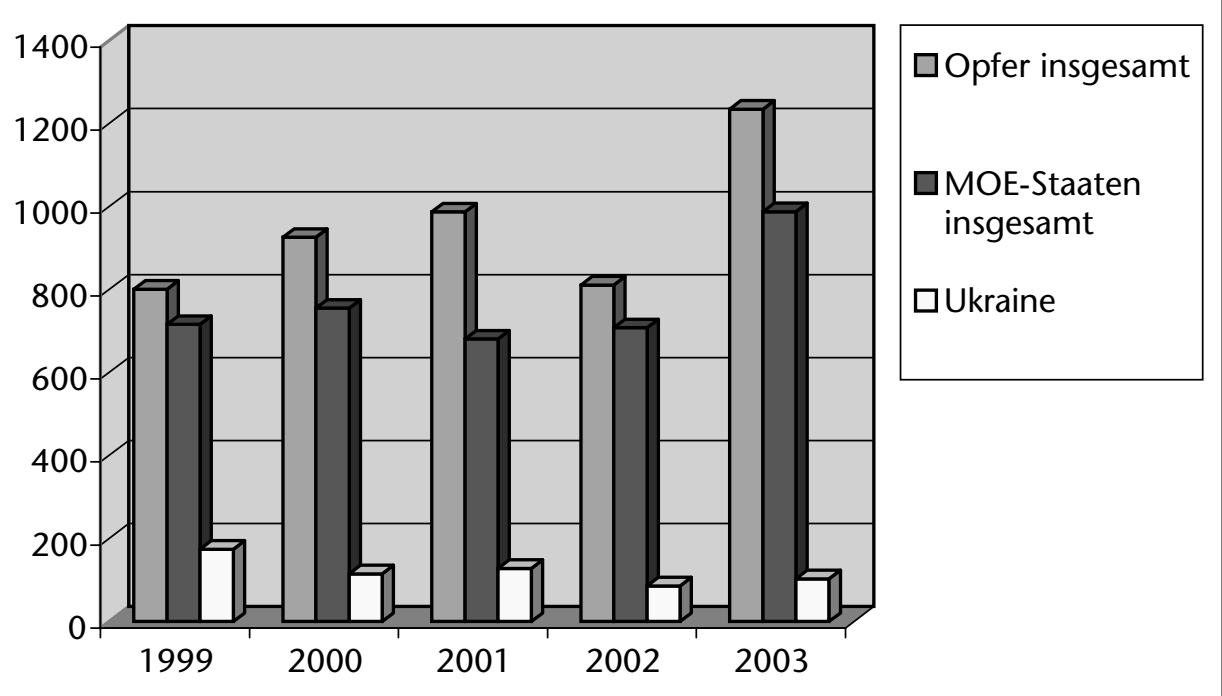

Quelle: BKA-Lagebild Menschenhandel

Als MOE-Staaten gelten: Bulgarien, Estland, Republik Jugoslawien, Lettland, Litauen, Republik Moldau, Polen, Rumänien, Russland, Slowakei, Slowenien, Tschechische Republik, Ukraine, Ungarn, Weißrussland. waren es nur etwa 150. Im Vergleich zu dieser marginalen Kriminalisierung ist sogar das klassische Delikt der ausbeuterischen Zuhälterei etwas bedeutsamer. Man kann daraus den Schluss ziehen, dass diese Art der OK-Ermittlungen primär ausländerrechtliche Zwecke erfüllt. Als »Banden- und Schleußerkriminalität« ist sie uns mittlerweile aus der Presse bestens bekannt, auch die Neigung der Politik, die »Opfer « zu instrumentalisieren. Ich fürchte, auch die Neuregelung wird daran wenig ändern, denn auch bei den leicht nachweisbaren Gewaltdelikten sind die Verurteilungsquoten niedrig. Daraus den Schluss zu ziehen, schwerer Menschenhandel sei ein Problem des Dunkelfeldes, ist ebenfalls wenig plausibel, da im Dunkelfeld typischerweise diffuse Zwangslagen anzutreffen sind. Diese scheinen aber geringer zu werden, wenn Prostituierte mehr Rechte haben.

Dies zeigt nicht zuletzt die Visa-Affäre. Schien es etwa zunächst, als befördere eine laxe VisaPraxis (wie seit 1999 etwa in Kiew) Phänomene wie »Frauenhandel«, so zeigen die Daten des polizeilichen Hellfeldes genau das Gegenteil. Strenge ausländerrechtliche Kontrollen schaffen zwar eine eigene "Kriminalität", aber keine erhöhte sozialschädliche Auffälligkeit der als Touristen eingereisten Menschen. Da alle eingereisten UkrainerInnen namentlich bekannt sind, hätten die LK-Ämter Daten gemeldet, wenn sie eine erhöhte Kriminalität dieser Gruppe festgestellt hätten. Dies ist aber gerade nicht geschehen. Verwunderlich ist dies nicht, sind doch auch sonst Einwanderer der ersten Generation eher unauffällig1.

Der bloße Verstoß gegen das Ausländergesetz ist somit kriminologisch rein formal und betrifft mehr oder weniger ausschließlich staatliche Interessen und völkerrechtliche Vereinbarungen. Etikettierungen als »kriminell « sind formal und bedeuten lebenspraktisch allenfalls Angst vor Abschiebung.

Auch der zweite Mythos ist nicht belegbar, wonach insbesondere osteuropäische Prostituierte ihre Dienste nur unter Zwang anbieten. Auch dieses Phänomen ist eher selten. Zwar gibt es ausreichende empirische Belege dafür, dass alle weiblichen Prostituierten häufig von Freiern belästigt und insgesamt häufiger Opfer von Gewalt als alle anderen Frauen sind, vermutlich häufig Opfer von Erpressung 2 . Aber es gibt keine belegbaren Kenntnisse darüber, dass insbesondere die illegal hier arbeitenden Prostituierten in 
die Prostitution gezwungen werden. Alle offiziellen Zahlen zeigen, dass die polizeilich deklarierten Tatverdächtigen und Opfer und die nach der Rechtspflegestatistik Verurteilten so erheblich differieren, dass Zweifel angebracht sind Selbst beim klarer konturierten Gewaltverbrechen des schweren Menschenhandels haben wir keine nennenswerten Verurteilungsquoten. Zwar kann man aus Hellfelddaten nicht allzu viel schließen, aber noch weniger aus vereinzelten Opfergeschichten, die sich als Elendsgeschichte lesen. Jedenfalls waren in der Vergangenheit zur Bekämpfung von Erpressung, Nötigung und Ausbeutung von Prostituierten Sondertatbestände eher kontraproduktiv. Wieso sollte sich das mit der Neuregelung ändern. Befragt man ferner vor Ort handelnde Polizeibeamte, also nicht die im politischen Raum aktiven Funktionäre, dann schildern diese die spezifischen Zwangssituationen der deklarierten Opfer eher als Konstellationen der Erpressung und gerade nicht als Zwang zur Prostitution. Unerfahrene Prostituierte würden mit »imaginären Forderungen « (so die von Marco Carini in der TAZ-nord am 26.04.2005 befragten Hamburger Polizeibeamten) in die Enge getrieben. Um sie zu begleichen, müssten sie wesentlich mehr arbeiten als zumutbar ist. Dies ist nicht verwunderlich. Schwarze Märkte sind noch patriarchalischer organisiert als der schlimmste Risikokapitalismus, und das sexuelle Dienstleitungsgewerbe ist seit je gewalttätig organisiert. Regulierte Märkte hingegen kennen Korrektive: organisierte Arbeitgeber, Vermieter, Vermittler auf der einen und organisierte Arbeitnehmer und Mieter auf der anderen. Beide Parteien sind ausgestattet mit guten Beratern und einem fein dogmatisiertem Rechtsschutz. In dieser bis 2002 illegalisierten Branche hingegen können Männer, die weit weg sind von zivilisierten Umgangsformen, ein gut eingeübtes (und der täterorientierten Kriminologie gut vertrautes) Verhaltensmuster ausleben. Das Ergebnis ist aber keine Zwangsprostitution, sondern ein Markt, in dem insbesondere junge und/oder ausländische, aber auch deutsche Prostituierte permanent übervorteilt, unter Druck gesetzt und langsam aber sicher wirtschaftlich ruiniert werden. Dies ist aber nur vereinzelt als Menschenhandel und ausbeuterische Prostitution/Zuhälterei verfolgbar, sondern Folge der fehlenden zivilrechtlichen Kontrolle.

Dem entsprechen die Daten des Hellfeldes. Selbst der BKA-Lagebericht zum Frauenhandel geht davon aus, dass weit mehr als $90 \%$ der deklarierten Opfer sich freiwillig (d.h. ohne Zwang) prostituieren. Die Verurteilungszahlen der offiziellen Rechtspflegestatistiken weisen in dieselbe Richtung. Strafgerichte verurteilen auch in diesen 5-10\% der Fälle, in denen Staatsanwaltschaften den Straftatbestand des schweren Menschenhandels oder der ausbeuterischen Prostitution bzw. Zuhälterei bejaht haben, eher selten. Wegen ausbeuterischer Prostitution etwa wur- den im Jahre 2002 nur 57 Täter bestraft, obgleich 547 ermittelte Tatverdächtige geliefert wurden, ähnliche Relationen haben die insoweit vergleichbaren Delikte des Frauenhandels. Die meisten Prostituierte können sich nämlich - so beurteilen die Gerichte die Aktenlage - kurzfristig von den für sie ungünstigen faktischen Vertragsverhältnissen lösen. Ihr Problem, so meine Schlussfolgerung, ist nicht der persönlich ausgeübte Zwang, sondern die Zwänge des unkontrollierten Marktes. Sie finden einfach keine besseren Arbeitsbedingungen, also lassen sie sich auf die vorgefundenen ein. Verstärkt wird diese ungünstige Ausgangssituation durch die Tatsache, dass es keine Institution gibt, weder Huren-Beratungsstellen noch Organisationen wie Verdi, welche eine aktive Gewerkschaftspolitik machen und mit anwaltlicher Unterstützung eine Verbesserung der Arbeitsbedingungen dieser (schein)selbstständig und unselbstständig tätigen Dienstleistenden erzwingen. Es dominiert ein Schulterschluss zwischen oft kirchlichen Beratungsstellen, Polizei und an Sozialarbeit angelehnter Beratung.

\section{Moralkampagnen}

Zum ersten mal wurde das Stichwort Zwangsprostitution populistisch platziert, als es darum ging einen »Moralisten an den Pranger « zu stellen (so der Stern Nr. 26 vom 18.06.2003 zum Ermittlungsverfahren gegen Friedmann wegen Kokainkonsums mit Prostituierten ${ }^{3}$ ). Rumänische Prostituierte hatten sich als Opferzeuginnen ${ }^{4}$ zur Verfügung gestellt und die Berliner Ermittlungsbehörden spielten kräftig mit und bedienten den politisch-publizistischen Verstärkerkreislauf um sex \& crime. Es folgte das von Oberstaatsanwalt Bülles in Köln mit Eifer betriebene und von Leyendecker in der SZ unkritisch kommentierte »Banden- und Schleußerverfahren« und die sich anschließende Visa-Affäre. Dieses Mal standen keine Moralisten am Pranger, sondern mit Joschka Fischer und seinem Staatssekretär Vollmer zwei beliebte Politiker und die Regierungsfähigkeit der rot-grünen Koalition. Wie wir heute wissen, hatte das Ganze mit Beförderung von "Zwangsprostitution « nichts zu tun, sondern eher damit, dass sich die Spitze des Auswärtigen Amtes im Dickicht zweier überbürokratisierter Behörden verfangen, welche konkret vor Ort schlecht zusammenarbeiten konnten. Aus Gründen, die hier dahinstehen müssen, gelang es den Polizeibehörden der Länder und dem Innenministerium durch Gesten der Empörung unbehelligt zu bleiben; denn die Umsetzung ausländerrechtlicher Vorschriften ist die originäre Aufgabe der Polizei. Aber das alles wissen wir erst heute - spätestens nach der Anhörung der Zeugin Klara Hoppmann, Beamtin des gehobenen Dienstes und Leiterin der Visa-Stelle in Kiew (Christian Füller TAZ vom 3.05.2005) ist klar, wo die Probleme ihren Anfang nahmen. Ihre Informationen waren den
Beamten des höheren Dienstes in Berlin nicht brisant genug, um sofort den direkten Kontakt mit dem gehobenen Dienst des jeweils anderen Hauses - dem Innenressort und den gehobenen Beamten der Visa-Stellen - herzustellen. Auf diese Weise geriet das Thema nicht auf den Radarschirm der beiden für das Innenressort und die Außenpolitik zuständigen Minister, sondern blieb auf der Abteilungsleiter-Ebene hängen. Politisch-publizistisch ließ sich dann aber dieses eher banale Bürokratieproblem aufblasen zu einem immer intensiver "gefühlten Skandal« (so zutreffend aber recherchefrei, was für gefühlte Skandale typisch ist, Bettina Gaus in der TAZ vom 7.03.2005). Im folgenden soll diesen sich selbst verstärkenden Kreisläufen des Nicht- oder Halbwissens eine weitere Dimension hinzugefügt werden.

Meine These lautet: Menschen- und Frauenhandel lässt sich erst dann - als ultima ratio auch mit strafrechtlichen Mitteln - verarbeiten, wenn ein arbeits-, miet- und zivilrechtlicher Unterbau geschaffen ist. Die Lage von Prostituierten bzw. von Niedriglohnabhängigen lässt sich nicht mit Kapitalismus kritischen Sprüchen und schon gar nicht mit dem moralistischen Entsetzen über »die Ware Frau « verbessern. Es ist aus meiner Sicht nichts gegen eine opferorientierte Strafverfolgung einzuwenden, wenn realistisch eingeschätzt wird, was Opferschutz bedeuten kann. Ein laienhafter Glaube an die opferschützende Kraft des Strafrechts ist für konkret Betroffene eher schädlich, da Strafrecht allenfalls symbolisch Opfern und der Allgemeinheit Genugtuung verschaffen kann. Lebenspraktisch hilft man konkret Betroffenen nur dann weiter, wenn man sie in die Lage versetzt, eigene zivile Rechte (im Zusammenhang mit oder außerhalb des Strafverfahrens) zu mobilisieren. So gesehen hat das ProstG lediglich eine Chance eröffnet, welche nicht genutzt wurde und vertan wird, wenn die derzeitige halbherzige Frauen- und Kriminalpolitik weiter geführt wird.

\section{Was kann eine Gleichstellungspolitik gegen Frauenhandel erreichen und wie verhält sich dieses Ziel zu dem der Internationalen Organisationen für Migration}

Wer sich zum Stichwort Frauenhandel kundig machen will, stößt auf zahlreiche Publikationen zu Problemen der Immigration, etwa der Internationalen Organisation für Migration - IOM. Insbesondere innerhalb der EU gibt es eine starke Koalition ansonsten unterschiedlich denkender Gruppen, welche sich zwar vordergründig ein gemeinsames Ziel gesetzt haben, nämlich Menschenhandel in jeder Form energisch zu »bekämpfen«. Aber sie blenden den naheliegenden ersten Schritt aus: die Verbesserung der Lebens- und Arbeitbedingungen legal im Sexgewerbe arbeitender Menschen. Stattdessen do- 
minieren unrealistische Kampfansagen, ein für die Kriminologie nicht gerade neues Phänomen. Um was geht es also und wie sollen die Gegenstrategien aussehen?

\section{Konturloses Strafrecht, kontraproduktiver Jugendschutz und Ermittlungsparagrafen}

Schon lange vor der Visa-Affäre bestand Grund sich über das vorhandene konturlose Strafrecht in den $\S \S 180$ b, 181 StGB alt (1993 - 2005) zu ärgern. Aber immerhin konnte man diese Paragrafen im Lichte des später erlassenen Prostitutionsgesetzes (2002) restriktiv interpretieren. Nach der Reform und Neufassung als $\S 232 \mathrm{ff}$ StGB im Februar 2005 fällt dieses Argument schwerer, weil die Gesetzgebung in Kenntnis der Legalisierung nun erneut die schlichte Vermittlung in die Prostitution, d.h. in eine legale Tätigkeit unter Strafe stellt, ohne dass erkennbar wird, worin eigentlich das strafwürdige Unrecht liegen soll (deshalb erörtern in diesem Heft Frommel/Schaar die strafrechtliche Frage genauer, insbesondere ob bereits das schlichte "Ausnutzen einer Zwangslage « - so der Grundtatbestand des § 232 StGB - ein bestimmbares strafwürdiges Verhalten sein kann, mit negativem Ergebnis). Birgit Thoma hingegen schildert die Reform eher positiv und hält sie nur in Randbereichen für kritikwürdig, würde also gegen die hier vertretene Intention - eher "mehr vom selben « vorschlagen und sogar weitergehende Wünsche zumindest der BadenWürttembergischen CDU empfehlen. Sie vermutet also mit der Mehrheit der Befürworter dieser Änderungsgesetzgebung, dass tendenziell jede "gehandelte Frau" eher ein Opfer ihrer Verhältnisse sei, während meine These dahin geht, die Probleme im unzureichenden arbeitsund mietrechtlichen Schutz für legale sexuelle Dienstleitungen (und dann natürlich erst recht für illegal hier arbeitende Menschen) zu sehen. Dahinter steht die Annahme, dass die Wahl des Berufs einer oder eines Prostituierten lediglich eine riskante Entscheidung ist, die nach den Maßstäben des Prostitutionsgesetzes sozialstaatlich abgefedert werden kann. Empfohlen wird daher bei ökonomisch ungleichgewichtigen (meist faktischen) Vertragsverhältnissen eine zivilrechtliche Inhaltskontrolle über $\S 134$ BGB (Nichtigkeit wegen gesetzwidriger Vereinbarungen) und über arbeits- und mietrechtliche Instrumente. Außerdem macht es Sinn, § 266 a StGB zu nutzen, da diese Strafnorm auch die Pflicht von Bordellbesitzern und Betreibern strafrechtlich implementieren könnte. Die meisten Prostituierten sind allenfalls scheinselbständig. Die Bordellbetreiber müssten nicht nur Steuern und Abgaben abführen, sondern sie machen sich - weit über das Steuerstrafrecht hinaus - strafbar, weil sie nicht nur diese ihre Pflicht verletzen, sondern ihre Mieten so kalkulieren, dass auch die Prostituierte selbst gar nicht mehr in der Lage ist, noch mehr Abzüge wirtschaftlich zu verkraften. Dies ist der "Zwang", der im ach so beliebten Stichwort "Zwangsprostitution « eher moralistisch verkleistert als benannt wird. Ausgangspunkt der hier angestellten Überlegungen ist daher der in der Überschrift zu $\S 180$ a und in 181 a StGB verwendete Begriff der »Ausbeutung«. Gesetzlich verboten ist danach schon vor dem strafrechtlichen Zugriff jede Form der ausbeuterischen Prostitution (»wer es unternimmt « ....) und ausbeuterische Zuhälterei. Nimmt man noch den bei imaginären Forderungen besser umsetzbaren Wuchertatbestand hinzu und legt den Maßstab des § 291 StGB zugrunde, ein krasses Mißverhältnis von Leistung und Gegenleistung, dann wäre bei einer zivilrechtlichen Inhaltskontrolle lediglich zu prüfen, was die Leistung und was die Gegenleistung war und ob diese in einem noch tolerierbaren Verhältnis zueinander stehen. So argumentiert jedenfalls die Rechtsprechung zu Wucher ${ }^{5}$. Zwar sind auch aus meiner Sicht Strafverfahren wenig sinnvoll, weil die betroffenen Prostituierten lediglich strukturell unfreiwillig, aber nicht in einem beweisbaren Sinne »unfrei = gezwungen in ihre ökonomische Isolierung geraten sind, so dass $\S \S 180$ a, 181 a StGB tendenziell nicht zu einer Verurteilung führen und auch der Wuchertatbestand des § 291 StGB häufig zu grob sein wird. Aber wie sieht es mit der gewaltsamen Durchsetzung nicht zurecht bestehender Forderungen aus? Erpressung und präventiver zivilrechtlicher Rechtsschutz wären zu aktivieren. Erst dann ließe sich beurteilen, ob die Einwilligung der Prostituierten in ungünstige Verträge relevant ist. Schließlich lassen sich objektive Maßstäbe finden wie Vergleichsmieten bzw. vergleichbare Regeln in anderen Branchen. Derartige Analogieschlüsse verbietet das Strafrecht, aber nicht das Zivilrecht. Dass Strafrecht dennoch immer wieder als Instrument gefordert wird, hängt damit zusammen, dass Laien sich notorisch Illusionen machen über dieses Rechtsgebiet. Seit der Abschaffung des Sittenwidrigkeitsverdiktes kann auch zivilrechtlich das zu viel Geleistetes zurückverlangt werden (da § 817 BGB nicht mehr entgegen steht und Prostituierte nicht mehr in den früher anzunehmenden illegalen Pakt mit ihren Vermietern, Arbeitgeber, Zuhältern zwingt).

Aber ist es realistisch, ausgerechnet auf das Zivilrecht $\mathrm{zu}$ hoffen? Anwaltskontakte sind schließlich das, was diese »Opfer « gerade nicht haben und was in dieser Branche weltfremd $\mathrm{zu}$ sein scheint. Aber wieso nutzen Beratungsstellen ihre Professionalität nicht? Wieso denken sie nicht über langfristig angelegte rechtliche Strategien nach? Wenn es um die Verschärfung von Strafrecht geht, sind sie doch präsent. Wer Zivilgerichte mobilisieren möchte, muss Glasperlenspiele vermeiden. Klar ist, dass insbesondere illegal hier arbeitende Menschen eine Zivilklage eher nicht erfolgreich durchstehen können. Da- her sollten sich Beratungsstellen an den legal hier arbeitenden Prostituierten orientieren und eine Stärkung ihrer zivilen Rechte empfehlen, also das ProstG implementieren statt mit unrealistischen ausländerrechtlichen Forderungen aufzuwarten.

\section{Autonomie und Gewalt - Zwang und Erpressung im Prostitutionsgewerbe}

Zweifellos gibt es im Prostitutionsgewerbe viel Gewalt und Zwang. Sie treffen aber die dort Tätigen höchst unterschiedlich. Besonders stark betroffen sind die jungen Straßenprostituierten ${ }^{6}$. Insbesondere Drogenabhängige setzen sich auf dem Straßenstrich erhöhten Gefahren aus. Derartiges könnte vermieden werden, wenn es nicht eine Reihe von undurchdachten Jugendschutzbestimmungen gäbe, welche einzeln und in ihrer Summe eher kontraproduktiv wirken, da sie nicht nur eine akzeptierende Sozialarbeit mit diesen jungen Menschen erschweren, sondern diese überdies auf den Straßenstrich zwingen, da die "Wohnungsgewährung « an unter 18 Jahre alte Prostituierte ohne Ausnahme verboten ist. Nun könnte dies ein kleiner Stein in einer vernetzten Prävention sein. Aber davon kann keine Rede sein. Nicht einmal die Schutzaltersgrenze - 16 Jahre - in § 182 StGB wird ernst genommen. Die bis zu diesem Alter längst normierte Freierbestrafung wird nicht umgesetzt. Ich schließe daraus, dass auch die neuerdings erhobene Forderung nach einer Bestrafung der Kunden von sog. "Zwangsprostituierten « symbolisch gemeint ist, da alle Kundigen wissen, dass diese Maßnahme zum Opferschutz ungeeignet ist.

Was also sind "Zwangsprostituierte «? Nehmen wir die im Spiegel 6/2005 S. 29 kolportierte Geschichte. Die Rede ist dort von Irina aus Russland, also einem Nicht-EU-Land, aus dem Irina nur als Touristin einreisen kann. Geschildert wird sie als »klein und dünn«. Die Bemerkung "sie hustet viel« schildert sie als zerbrechlich. Sie sei mit einem erschlichenen Visum, für das sie 450 Dollar zahlen sollte, das dann aber 650 Dollar gekostet hat, nach Deutschland gekommen. "Inzwischen schuldet sie den Leuten, die sie in die Bundesrepublik verfrachteten, 5500 Euro. Zu den Kosten für die Schleusung kommen Miete, Verpflegung, die Fahrten zu den Freiern ...« hinzu. Es folgen Drohungen für den Fall, dass sie nicht zahle. Der Zwang beruht auf imaginären Schulden. Ihre Gläubiger könnten kein Zivilgericht einschalten, da es für derartige Forderungen keinen Rechtsgrund gibt. Aber auch die Schuldnerin kann sich faktisch nicht wehren, wenn sie hier illegal lebt und deshalb jederzeit ausgewiesen werden kann.

Kein Wunder, dass die Beratungsstellen versuchen ihren Klientinnen vorübergehende Aufenthaltsgenehmigungen zu verschaffen. Mög- 
lich ist dies, wenn diese sich als Opferzeuginnen in einem Strafverfahren wegen Frauenhandel zur Verfügung stellen. Die EU hat sogar eine entsprechende Richtlinie erlassen mit dem geradezu absurden Titel: »EU-Richtlinie des Rates über die Erteilung kurzfristiger Aufenthaltstitel für die Opfer der Beihilfe zur illegalen Einwanderung und des Menschenhandels, die mit den zuständigen Behörden kooperieren «, verabschiedet am 29.4.2004 (RL 2004/81/EG, ABl.L 261/19 v. 6.8.2004).

Das Problem ist nur, dass Gegenstand in diesen Verfahren nicht die Erpressung der sich hier illegal aufhaltenden Migrantinnen ist, sondern ausländerrechtliche Probleme. Die Zeuginnen werden nicht in ihrer Angelegenheit unterstützt, sondern eher zum Mittel zur Umsetzung staatlicher Interessen eingesetzt. Da sie sich meist nicht gegen ihren Willen prostituieren, sondern lediglich erpressbar waren und meinten, ihre imaginären Schulden abzahlen zu müssen, ist der Umweg über immer weiter gefasste Strafbestimmungen gegen Menschenhandel nicht nur umständlich, sondern kontraproduktiv. Die deklarierten Opfer legitimieren zwar die Strafverfolgung, aber sie bestimmen nicht die Gestaltung des Strafverfahrens. Was wäre denn ein realistischer Ausweg?

Am besten beginnen wir bei den üblichen Vorurteilen, breit dargetan in derselben Ausgabe des Spiegel 6/2005 S. 52: Luftnummer im Puff. Danach sei das von grünen Politikerinnen ausgeklügelte ProstG vom 1.01.2005 allenfalls dafür gut, eine kosmetische Verbesserung der Arbeitsbedingungen in Häusern wie dem Arabella-Eros-Center zu erreichen, etwa eine Kaffemaschine auf dem Flur oder ein Christbaum zu Weihnachten. So etwas habe früher als Förderung der Prostitution verfolgt werden können, nun sei derartige Imagepflege legal (zitiert wird im Spiegel der Geschäftsführer, Herr Bunsen aus Frankfurt a.M.). Die Mieten für ein kleines Zimmer von 100-150 Euro pro Nacht werden kurz erwähnt. Auf die Idee, dass dies Mietwucher sein könne, kommt der Redakteur nicht, aber er macht sich lustig über Gutmenschen, welche glaubten bei solchen Mieten hätten die Damen noch Lust auf Steuern und Sozialversicherungsabgaben: eine Luftnummer eben sei der Versuch der Legalisierung. Ich frage mich, wieso sich die örtliche Polizei, das Landeskriminalamt, die Beratungsstellen, die Finanzämter und die AOK nicht zusammensetzen, um Steuern und Sozialversicherungsabgaben von Herrn Bunsen nachzufordern, in Anrechnung auf die Wuchermiete. Dann könnten in Zukunft zivilisierte Verhältnisse dort einkehren und die osteuropäischen Migrantinnen im erweiterten EU Raum nach Ablauf der Übergangsfristen auf bessere Arbeitsbedingungen hoffen. Bis es so weit ist, könnten sie aber auch in ihren »selbstständigen« Beschäftigungsverhältnissen dem Beispiel der Damen im Arabella-Eros-Center folgen. Noch zeichnet sich nichts derartiges ab.

Fragen wir aber dennoch, warum nicht und wieso etwa Verdi sich dieser Arbeitnehmerinnen so gut wie nicht, zumindest nicht ernsthaft, angenommen hat. Die in den letzten Jahren wieder stärkere Fixierung der Politik auf das Strafrecht, noch schlimmer: auf verkapptes Ausländerrecht, verstärkt das Desaster. Eine der juristischen Beraterinnen der Grünen, Margarete von Galen, kommentiert zwar das ProstG und gibt kluge Ratschläge, aber die versierte Strafverteidigerin hat sich nicht - bei allen Verdiensten, die sie sich mit diesem Buch erworben hat - überlegt, wie man die Lage der legal in Deutschland arbeitenden (erwachsenen) Prostituierten verbessern könnte. Sie schaut auf die Strafrechtsnormen, liest in den $\S \S 180$ a, 181 a StGB (verbotene Ausbeutung durch Bordellbetreiber und Zuhälter) das Wort »Ausbeutung « und konstruiert eine positiv zu bewertende Veränderung der Sicht auf Prostituierte früher und heute. »Nach alter Vorstellung « (so die Autorin in »Rechtsfragen der Prostitution «, Rdnr. 341 - 342) habe eine zugeschriebene Persönlichkeitsstruktur verhindert, Prostituierte als frei entscheidende Personen anzusehen. Heute sei dies Rechtsgeschichte. Daher müsse man die von ihnen geschlossenen Verträge auch so, wie sie nun einmal sind, akzeptieren. Ich bin mir nicht sicher, dass dies die richtige Konsequenz ist. Strafrechtsdogmatisch geht die Verfasserin von der zutreffenden Annahme aus, dass im Strafverfahren eher ein Freispruch für Bordellbetreiber/Zuhälter zu erwarten ist. Das ist nicht zu beanstanden. Aber zivilrechtlich macht ihre Annahme keinen Sinn. Vertragsautonomie ist ein hoher, aber nicht der einzige Wert. Das Fehlen jeder gerichtlichen Kontrollen für extrem überhöhte Forderungen, insbesondere extrem überhöhte Mieten, die Zulässigkeit von "Strafgelder « bei zu seltenen Kundenkontakten etc. zwingt die Prostituierte zwar nicht zu der von ihr gewählten Tätigkeit (Zwangsprostitution), sie kann meist aussteigen, aber es kann doch nicht Sinn des ProstG sein, lediglich das Ziel der Hilfe beim Ausstieg zu erreichen. Es widerspricht allen Grundprinzipien einer sozialen Marktwirtschaft, abhängig oder scheinselbständig Arbeitenden nur die Wahl zu lassen: Verschuldung oder Ausstieg.

Die meinetwegen strafrechtlich vertretbare weit gefasste Rechtfertigungslösung verkennt die Bedeutung der $\S \S 180$ a, 181 a StGB als Verbotsnormen zur Kontrolle von zivilrechtlichen Verträgen (auch faktischen Vertragsverhältnissen) über $§ 134$ BGB.

Nach der im Zivilrecht völlig unbestrittenen Rechtsprechung kommt es bei der Frage der Nichtigkeit von Verträgen nach § 134 BGB lediglich darauf an, ob der Sinn einer Verbotsnorm der Schutz einer Vertragspartei vor solchen Geschäften ist. Wenn das Verbot der Ausbeutung einen Sinn haben soll, dann doch den, Wuchermieten zu unterbinden. Wo die Grenze anzusetzen ist, kann hier aber nicht pauschal beantwortet werden, da jede Richtigkeitskontrolle nur konkret erfolgen kann. Aber unerlässlich ist ein effektiver Schutz von Prostituierten vor wucherischen Verträgen, und zwar nicht nur vor einer Ausbeutung i.S. einer gezielten Verarmung, sondern gegen alle Formen der krassen wirtschaftlichen Übervorteilung. Selbst wenn strafrechtlich eine rechtfertigende Einwilligung vorliegen sollte, genügt es zivilrechtlich, wenn Geschäfte dieser Art grob unbillig und tatbestandsmäßig sind, auf die Frage der Rechtfertigung kommt es für § 134 BGB nicht an. Ausbeuter müssen nicht strafbar handeln im Sinne einer rechtswidrig und schuldhaft begangenen Tat. Es genügt, wenn sie Forderungen durchsetzen wollen, welche krass unangemessen sind. Die Prostituierte kann im übrigen nicht nur für die Zukunft, sondern auch rückwirkend erhebliche Summen zurückfordern bzw. Bordellbesitzer zwingen, rückwirkend Steuern und abgaben zu bezahlen. Alle entgegen stehenden Absprachen und Gepflogenheiten sind wegen $\S 134$ BGB nichtig und über das Bereicherungsrecht rück abzuwickeln. Da die Nichtabführung von Steuern und Sozialabgaben außerdem ggf. nach § 266 a StGB strafbar ist, kann auch eine eindrucksvolle Drohkulisse aufgebaut werden statt den konturlosen »Menschenhandelsparagrafen « zu bemühen. Sowohl der Fiskus als auch die Sozialversicherungsanstalten sind somit gut beraten, streng genommen müsste sie der Rechnungshof dazu verpflichten, die zivilrechtlichen Strategien von Beratungsstellen (im Schatten ebenfalls möglicher Strafverfahren) aktiv zu unterstützen und eine Art runden Tisch zu bilden, damit man sinnvoll und präventiv effektiv vorgehen kann. Erst ganz am Ende stünde dann das, was zurzeit als prima ratio (auch im Artikel von Birgit Tho$m a$ ) empfohlen wird, die ausländerrechtliche Karte bei den illegal Beschäftigten. Wie immer man diese Karte ziehen möchte: sie hilft den illegal hier arbeitenden und um ihren Lohn betrogenen und erpressten Prostituierten am wenigsten, während zivilrechtlich operierende Beratungsstellen diesen Wirtschaftssektor langsam aber allmählich sanieren könnten. Die zurzeit besonders ausgegrenzten Migrantinnen sollen spezialisierte Beratungsstellen vorfinden. Aber grundsätzlich sollten sich Beraterinnen von dem Gedanken verabschieden, dass ihre Aufgabe primär eine psychosoziale sei und die beste Lösung der Ausstieg aus der Prostitution. Dies zu entscheiden ist Sache derer, die solche Dienstleistungen leisten. Professionelle Beratung sollte gewerkschaftlich denken und effektive Wege der Verbesserung der Arbeitsbedingungen anbieten. Dies geht aber sicher nicht über Strafrecht pur, sondern allenfalls über § 134 BGB i.V.m. den einschlägigen Strafnormen. Wo viel verdient wird, funktioniert eine zivilrechtliche Kontrolle, jedenfalls besser als abenteuerliche Konstruktionen wie Vermögensstrafen, Verfall und erweiter- 
ten Verfall in Strafverfahren, die letztlich ausländerrechtliche Ziele verfolgen.

\section{Fußnoten:}

1 So auch Christian Pfeiffer, Kriminalistik 2005, 217, allerdings mit anderen Berechnungen.

2 Wer nichts gegen den weiten Gewaltbegriff von Ursula Müller in der vom BMFSFJ am 24.09.2004 vorgestellten Gewaltstudie hat, kann im Internet in der Zusammenfassung (S.24) nachlesen, dass Prostituierte und inhaftierte Frauen mit $92 \%$ gegenüber $58 \%$ (Hauptstudie) durchgängig angeben, in ihrem Leben bereits sexuell belästigt worden zu sein. Auch die Gewalterfahrung ist deutlich höher gegenüber der übrigen weiblichen Bevölkerung, was wenig verwunderlich ist, aber angesichts der erheblichen Differenz dennoch aussagekräftig ist.

Wer es genauer wissen will, wird zwischen jungen und älteren Prostituierten unterscheiden und erkennen, dass insbesondere die Jugendlichen auf dem Straßenstrich viel Brachialgewalt erleben, während erwachsene Prostituierte in entsprechenden Wohnungen ihr Leben sehr viel geregelter organisieren können, vgl. Leopold/Grieger, Das Parlament vom 20.12.2004 B 52 - 53/2004. Ein entsprechendes Forschungsvorhaben von Kavemann/Leopold, von den GRUNEN in Auftrag gegeben, geht dem noch genauer nach.

3 »Das war doch widerlich und gehört bestraft « kommentierte Siegfried Kauder und initiierte einen CDU-
Gesetzesentwurf zur sog. Freierbestrafung, SZ vom 25.01.2005.

4 Nur dann können sie eine befristete Aufenthaltserlaubnis erhalten.

5 Fischer, Tröndle/Fischer $\S 180$ a Rdnr. 27 überträgt diese Rechtsprechung neuerdings auch auf die ausbeuterische Prostitution und Zuhälterei, vgl. ferner § 291 Rdnr. 17. Die Kommentierung in diesem praktisch bedeutsamen Kommentar ist seit der 52 . Aufl. 2004 ausgesprochen offen für derartige Probleme, was zeigt, dass die Rechtsprechung eher fortschrittlicher ist als die oft populistische Gesetzgebung.

6 Beate Leopold/Elfriede Steffan, Evaluierung unterstützender Maßnahmen aus der Prostitution (EVAProjekt). SPI-Forschungs-GmbH, Berlin 1997.

\title{
Einwände gegen den am 19.02.2005 neu gefassten Straftatbestand des Menschenhandels in $\S 232$ Abs. 1 StGB
}

\author{
Monika Frommel und Martin Schaar
}

W er den an die Stelle des alten $\S 180 \mathrm{~b}$ StGB getretenen Auffangtatbestand des Menschenhandels liest, erkennt unschwer, dass hier weder ein Gewalt-, noch ein Täuschungsdelikt geschaffen worden ist, auch keine Spezialvorschrift zur Willensbeugung (wie Thoma in diesem Heft unterstellt), sondern eine Strafnorm, welche illegale Märkte kontrollieren will. Illegal sind diese Märkte aber in erster Linie wegen des Ausländerrechts. Kritisiert werden soll im folgenden nicht der gesamte Komplex der $\S \S 180$ b, 181 des alten und der seit 2005 in den $\S \S 232 \mathrm{ff}$ neu gefassten Tatbestände, sondern nur ein Ausschnitt: die Vermittlung von Nicht-EUBürgern und/oder jungen Frauen unter 21 Jahren in die Prostitution. Unberücksichtig bleiben hier also die $\S \S 232$ Abs. 4, 233 ff. StGB, deren Unrecht bis zum 19.02. 2005 im Verbrechenstatbestand des § 181 StGB (schwerer Menschenhandel) geregelt und nun lediglich mit Qualifikationen versehen worden sind, welche einerseits eine härtere, andererseits aber wegen der vorgesehenen minderschweren Fällen eine milde Bestrafung, sogar eine Informalisierung ermöglichen.

\section{$\S 232$ StGB (neu gefasst am 19.02.2005):}

(1) Wer eine andere Person unter Ausnutzung einer Zwangslage oder der Hilflosigkeit, die mit ihrem Aufenthalt in einem fremden Land verbunden ist, zur Aufnahme oder Fortsetzung der Prostitution oder dazu bringt, sexuelle Handlungen, durch die sie ausgebeutet wird, an oder vor dem Täter oder einem Dritten vor oder von dem Täter oder einem Dritten an sich vornehmen zu lassen, wird mit Freiheitsstrafe von sechs Monaten bis zu zehn Jahren bestraft. Ebenso wird bestraft, wer eine Person unter einundzwanzig Jahren zur Aufnahme oder Fortsetzung der Prostitution oder zu den sonst in Satz 1 bezeichneten sexuellen Handlungen bringt.

(2) Der Versuch ist strafbar.

Behandelt wird hier nur dieser Auffangtatbestand, der von der Gesetzgebung bewusst so weit wie möglich gefasst worden ist und jede Vermittlung von ausländischen und jungen Frauen und Männern unter 21 Jahren in die Prostitution bestraft. Ausübung von Zwang oder Täuschung ist hier - im Unterschied zu Abs. 4 nicht notwendig. Verfolgt wird also nicht nur die sog. Zwangsprostitution, sondern fast jede grenzüberschreitende Prostitutionsvermittlung. Setzt der Täter Gewalt, Drohung und List ein, dann wird er nach $\S 232$ Abs. 4 StGB erheblich härter bestraft (vgl. den Gesetzestext anbei). Zwar bestimmt der moralisch aufgeladene Begriff der Zwangsprostitution das Thema Menschenhandel, aber diese Assoziation ist vorschnell und irreführend. Kritisiert wird hier also nicht der in $\S 232$ Abs. 4 StGB normierte Verbrechenstatbestand des schweren Menschenhandels, der klare Täter-Opfer-Situationen und klare Tathandlungen beinhaltet (Gewalt, Drohung und List), schließlich wird die Ausübung von Zwang auch sonst strafrechtlich verboten, etwa als Erpressung, und Täuschungen kennen wir auch, allerdings überrascht die weite Tathandlung der List. Derartiges würde für Betrug nicht genügen. Auch haben wir es im Unter- schied zur ebenfalls sehr weit gefassten sexuellen Nötigung (vgl. die Urteilsanmerkung zu § 177 Abs. 1 Nr. 3 StGB - sexuelle Nötigung unter Ausnutzung einer schutzlosen Lage - in diesem Heft) beim oben zitierten Auffangtatbestand nicht mit einer individualisierten Täter-OpferBeziehung zu tun, sondern mit dem problematischen Versuch, jede Vermittlung in schwarze und graue Märkte zu sanktionieren und bei Gewerbsmäßigkeit sogar als Verbrechen (§ 232 Abs. 3 Nr. 3 StGB). Geschützt werden soll durch $\S 232$ StGB nicht das sexuelle Selbstbestimmungsrecht (dies war auch in $\S 180 \mathrm{~b}$ StGB nicht das Rechtsgut), sondern die wirtschaftliche und soziale Bewegungsfreiheit von Menschen, die in einer oft erst durch das Ausländerrecht geschaffenen Notlage und/oder einer unabhängig davon existierenden wirtschaftlichen Zwangslage » zu fast allem bereit« sind. Außerdem werden heranwachsende Prostituierte wie Jugendliche behandelt, also weitgehend von der Legalisierung sexueller Dienstleistungen ausgenommen.

Die Struktur eines solchen Tatbestandes unterscheidet sich erheblich von der des lange umstrittenen § 177 Abs. 1 Nr. 3 StGB (der sexuellen Nötigung unter Ausnutzung einer schutzlosen Lage), aber es gibt Gemeinsamkeiten. Immerhin benutzt die Gesetzgebung auch hier den Begriff des »Ausnutzens « einer Lage, die entweder mit einer erhöhten Schutzlosigkeit oder wie in § 232 mit einer Unfreiheit verbunden ist. Ähnlichkeiten bestehen auch zu der im übrigen so gut wie nicht praktisch umgesetzten Jugendschutznorm 